\title{
Las cooperativas y los jóvenes
}

\author{
Alejandro Martínez Charterina \\ Catedrático de la Universidad de Deusto \\ Director del Instituto de Estudios Cooperativos \\ Facultad de Derecho
}

Sumario: 1. Introducción. 2. Algunas características de los comienzos del siglo XXI. 3. Las cooperativas y su identidad. 4. Los jóvenes y las cooperativas. 5. Los jóvenes comparten los valores cooperativos. 6. Conclusión: una mirada al futuro. Bibliografía.

Resumen: En el momento presente, a comienzos del siglo XXI, los grandes cambios experimentados invitan al estudio y reflexión acerca de la situación de los jóvenes y su posición respecto a las cooperativas. Los jóvenes necesitan a las cooperativas tanto como éstas los necesitan a ellos. Resulta fundamental la investigación acerca de los valores que exhiben los jóvenes y su sintonía con los valores cooperativos que conforman su identidad.

Palabras clave: Cooperativismo y jóvenes.

Abstract: The current great challenges experienced in the early $21^{\text {st }}$ century invite to study and reflect on the situation of youth and their approach to cooperatives. Youth need cooperatives just as much as they need them. Research should be conducted on the values exhibited by young people and their consistency with the cooperative values that make up their identity.

Keywords: Cooperativism and youth. 


\section{Introducción}

La atención hacia los jóvenes ha cobrado fuerza en el ámbito cooperativo en los últimos tiempos.

El cambio acelerado y el avance de la globalización que se han dejado notar ampliamente desde la parte final del siglo pasado, así como la crisis profunda y prolongada que venimos padeciendo en los comienzos del presente, han acrecentado, con toda seguridad, esta necesaria atención.

Las cooperativas se distinguen de otros modelos de empresa por acomodar sus acciones a unos principios a través de los cuales exhiben unos valores que juntamente con ellos conforman su identidad.

Siendo esencial a las cooperativas su identidad cabe preguntarse en qué medida los jóvenes de hoy comparten entre sus valores aquellos que identifican a las cooperativas, considerando que estos jóvenes constituyen el futuro de las mismas, al mismo tiempo que es mucho lo que de ellas pueden recibir.

Desde estas consideraciones participé en la obra colectiva titulada Difusión de los valores y principios cooperativos entre la juventud ${ }^{1}$, que inspira notablemente el contenido del presente trabajo.

En el mismo me fijaré en esas notas características del momento presente de cambio y crisis, la relación de las cooperativas y los jóvenes, la presencia de valores cooperativos en buena parte de los mismos, a tenor de la última tipología de jóvenes y valores para España del año 2014, para terminar con unas consideraciones finales a modo de conclusión.

\section{Algunas características de los comienzos del siglo XXI}

Los procesos de desarrollo económico vividos desde que se inauguró el proceso de industrialización en la Inglaterra de la segunda mitad del siglo XVIII han llevado aparejados, entre otras muchas cosas, procesos de cambio en la sociedad, a partir del cambio tecnológico, cada vez más acelerados².

1 Alejandro Martínez Charterina, «Las cooperativas y los jóvenes en la actualidad», en Vega María Arnáez Arce (coordinadora), Difusión de los valores y principios cooperativos entre la juventud, (2015).

2 Precisamente una de las razones, seguramente la más importante, de la aparición tardía de la Ciencia de la Economía, a pesar de que la actividad económica que constituye su objeto de estudio haya acompañado a las personas siempre, reside en la lentitud 
Si la distancia generacional era notoria en el pasado, en la actualidad es bastante mayor como consecuencia del cambio acelerado, el estado de la globalización, y el particular momento económico.

Merece la pena detenerse un momento en el ensayo de Michel Serres $^{3}$ que presenta un estereotipo de joven que por efecto del cambio de las nuevas tecnologías queda unido a un teléfono móvil que le proporciona el conocimiento y la comunicación que precisa en todo momento.

Los jóvenes de hoy son muy distintos de las generaciones anteriores y su entorno es también muy diferente:

«Han nacido de forma programada, muchos de sus progenitores están divorciados, y viven en un ambiente multicultural entre religiones, lenguas y costumbres diversas.

Habitan un espacio virtual, en el que conocen de forma distinta y escriben y hablan de otra manera.

No saben vivir en pareja, se divorcian, no saben mantenerse quietos, se mueven, viajan, no rezan en la Iglesia.

El conocimiento está en la red y es accesible a todos.

En este proceso de cambio todo está por hacer. Se escucha la voz de todos y el saber se ha democratizado» ${ }^{4}$.

Junto al cambio acelerado tenemos que examinar la crisis que venimos padeciendo y sus efectos. Se viene considerando que desde la del 29 no se había conocido en Estados Unidos una situación de desempleo de larga duración como la actual. Naturalmente aunque las crisis se superan en la historia de la economía dejan profundas huellas en determinados colectivos, especialmente en aquellos que por su mayor edad no pueden reincorporarse al mercado laboral y en aquellos otros compuestos por los jóvenes que no pueden alcanzar un empleo que les permita independizarse y caminar hacia el futuro, haciéndoles perder oportunidades, de las que en otras circunstancias habrían disfrutado 5 .

del cambio, la consideración de la economía como una prolongación del medio físico. El proceso de industrialización aceleró el cambio y permitió el cuestionamiento necesario para que Adam Smith escribiera La riqueza de las naciones. Puede verse en Alejandro Martínez Charterina, Introducción a la economía, (2011), p. 19.

3 Michel Serres, Pulgarcita, (2014).

4 Síntesis del ensayo de Richard Serres recogido en Alejandro Martínez Charterina, o.c., (2015), p. 125.

5 En este sentido se posiciona el nobel de economía Paul Krugman, uno de los pocos que anunció la crisis sin ser escuchado, en su obra ¡Acabad ya con esta crisis!, (2012), ps. 18-22. 
Esta crisis financiera de los Estados Unidos se trasladó por el mundo globalizado, en particular por Europa, con toda facilidad, a través de los productos derivados, como tantas veces se ha relatado en estos años ${ }^{6}$.

La economía española, en la que a la crisis financiera se unió la explosión de la burbuja inmobiliaria, es una de las que más han sufrido esta crisis junto con los países periféricos de la Unión Europea. El retroceso de la actividad económica dejó tasas de paro de hasta el 26,94\% en el primer trimestre de 2013 , tiempo en el que la tasa de paro juvenil (menores de 25 años) era de 57,22\% ${ }^{7}$.

Aunque la economía española parece que comienza su recuperación, con tasas de paro correspondientes al tercer trimestre de 2015 de $21,18 \%$ y el $46,6 \%$ de paro juvenil ${ }^{8}$, quedan, como hemos mencionado anteriormente, colectivos que deberán afrontar la imposibilidad de tener un empleo el resto de su vida o que, y aquí puede situarse un gran colectivo de jóvenes, deberán acomodarse a situaciones de subempleo, emigración y precariedad, además de arrastrar la desilusión que sobreviene a la falta de oportunidades en el momento adecuado.

Pero, además de esto, debe considerarse como nota cualificada el hecho de que, además de crisis financiera, esta crisis es una crisis de valores, o, por mejor decir, de falta de valores ${ }^{9}$. En este sentido se expresa Leopoldo Abadía en todas las obras que ha publicado estos años de crisis, que se refiere reiteradamente a la falta de ética empresarial, crisis de decencia, corrupción y relativismo moral que acompaña este tiempo ${ }^{10}$.

No causa ninguna extrañeza desde esta perspectiva la diferencia de rentas entre los ejecutivos y operarios estudiadas para los Estados Unidos, cuyos abanicos salariales se han multiplicado por 13,5 en los úl-

6 Yo lo he referido con detalle en "Las cooperativas frente a la crisis», (2010), ps. 203-205.

7 Datos de la Encuesta de Población Activa del Instituto Nacional de Estadística. En números absolutos los parados eran 6.202.700, y los jóvenes parados 960.400 .

8 Según la Encuesta de Población Activa del Instituto Nacional de Estadística del tercer trimestre de 2015, el número absoluto de parados ha quedado reducido a 4.850.800, siendo los desempleados menores de 25 años 766.700.

9 Lo he analizado en Alejandro Martínez Charterina, o.c., (2015), ps. 122-123.

10 Leopoldo Abadía, La crisis ninja y otros misterios de la economía actual (2009), p. 133 ss.; La hora de los sensatos (2010), p. 79; ¿Qué hace una persona como tú en una crisis como esta? (2010), p. 167; El economista esperanzado (2012), p. 173 ss.; La economía en 365 preguntas (2013), ps.51, 181 y 182. 
timos 50 años $^{11}$, y que por extensión se pueden contemplar en otras economías con más o menos elementos correctores.

Y en el mismo sentido los informes Oxfam de los últimos años de este tiempo de crisis vienen denunciando que la austeridad económica de los países de Europa no sólo paraliza el crecimiento sino que es causa de desigualdad ${ }^{12}$, cómo crece la desigualdad económica en la mayor parte de los países del mundo ${ }^{13}$, cómo la riqueza del mundo se concentra en las pocas manos de una pequeña élite mundial que actúa como lobby para influir en los presupuestos públicos y la política fiscal ${ }^{14}$, y cómo la desigualdad extrema está alcanzando niveles insoportables, toda vez que el $1 \%$ de la población del mundo tiene en la práctica casi más riqueza que el otro $99 \%$ y dispone de paraísos fiscales de ocultación, de modo que la crisis actual se puede tipificar de crisis de desigualdad ${ }^{15}$.

La riqueza queda concentrada en pocas manos, el $1 \%$ poseía en 2014 el $48 \%$ de la riqueza del mundo, mientras un $80 \%$ detentaba el 5,5\%, con tendencia creciente ${ }^{16}$. El descenso inicial de la riqueza con la crisis en los más ricos se ha recuperado rápidamente y con cre$\operatorname{ces}^{17}$. Y seguramente la concentración de la riqueza será mayor en la medida en la que una parte de la misma descansa en los llamados paraísos fiscales.

Las élites, vinculadas a grandes empresas en sectores financieros, farmacéuticos y de salud, llevan a cabo una importante acción de lobby en favor de sus intereses ${ }^{18}$, lo que coincide con la consideración de los

11 La diferencia de renta entre ejecutivos y operarios en Estados Unidos «era de 24 a 1 en 1965; de 71 a 1 en 1989; de 262 a 1 en 2005 y de 325 a 1 en 2011 » nos informa Christian Felber en su libro La economía del bien común, (2012), p.11. Paul Krugman destaca el caso de los gestores de fondos de cobertura reunidos a cuarenta minutos en tren de la isla de Manhattan: «En 2006, los veinticinco administradores mejor pagados ganaron 14.000 millones de dólares: tres veces la suma de los sueldos de los ochenta mil maestros de escuela de la ciudad de Nueva York», o.c., (2012), p. 84.

12 Informe Oxfam, La trampa de la austeridad. El verdadero coste de la desigualdad en Europa, (2013), siguiendo las consideraciones de Joseph Stiglitz en este mismo sentido

13 Informe Oxfam, Gobernar para las élites. Secuestro democrático y desigualdad económica, (2014), p. 1.

14 Informe Oxfam, Riqueza: Tenerlo todo y querer más, (2015), p. 1.

15 Informe Oxfam, Una economía al servicio del 1\%. Acabar con los privilegios y la concentración de poder para frenar la desigualdad extrema, (2016), p.1.

16 Informe Oxfam, O.C., (2015), p. 2. Con datos actualizados de Credit Suisse 92 milmillonarios poseían en 2013 la misma riqueza que la mitad más pobre de la población del mundo (p.4).

17 Informe Oxfam, o.c., (2014), p. 5.

18 Informe Oxfam, O.c., (2015), ps. 7-8. 
ciudadanos, que manifiestan en una encuesta en ocho países (ocho de cada diez personas en España) su convicción de que las leyes están realizadas en favor de los ricos ${ }^{19}$.

Por esta vía se profundiza y perpetúa la brecha de desigualdad, y se deteriora el estado de bienestar ${ }^{20}$, llegando a amenazar la misma democracia política ${ }^{21}$.

\section{Las cooperativas y su identidad}

En este contexto actual de cambio acelerado y de profunda crisis económica, de valores y de desigualdad es en el que debemos situar a las cooperativas.

Hoy como ayer las cooperativas constituyen asociaciones de personas que tratan de afrontar algunas de sus necesidades a través de una empresa económica que se gestiona democráticamente ${ }^{22}$. En la cooperativa se une de forma indisoluble el elemento social, las personas que se asocian, y el elemento económico, la empresa a través de la cual alcanzan la satisfacción de sus aspiraciones ${ }^{23}$.

Las cooperativas «nacieron para dar respuesta a unas necesidades, se desarrollaron resolviendo sus propias crisis internas, y salieron a flote, ayer como hoy, de las crisis económicas» 24 .

Cuando los tejedores de Rochdale no pudieron conseguir un aumento de sus salarios, muy justificado por los abundantes beneficios de

19 Informe de Oxfam, O.c., (2014), ps. 10-11.

20 Informe de Oxfam, o.c., (2014), ps. 12 ss.

21 Informe de Oxfam, o.c., (2014), p. 2. De aquí las recomendaciones de los informes de referencia de 2015 y 2016 en el sentido de pagar salarios dignos reduciendo las diferencias abismales con las retribuciones a los altos directivos, fomentar la igualdad de las mujeres, controlar la influencia de las élites poderosas, garantizar el acceso a los medicamentos controlando los precios de los mismos, distribuir el esfuerzo fiscal justa y equitativamente, utilizar el gasto público para reducir la desigualdad, y hacer desaparecer los paraísos fiscales. (Pueden verse los informes de 2015, ps. 10-11, y de 2016, p. 8)

22 Así define la cooperativa la Alianza Cooperativa Internacional. I.C.A.: Declaración de la Alianza Cooperativa Internacional sobre la Identidad Cooperativa. Los principios cooperativos (1996), p. 17.

23 Georges Fauquet, «Le secteur coopératif», en Oeuvres, (1965), ps. 39-40. En el mismo sentido se refería José M. ${ }^{a}$ Arizmendiarrieta, inspirador y guía de las cooperativas de Mondragón, a ellas diciendo que «lo social debe acreditarse por lo económico no menos que lo económico debe autentificarse por lo social», en "La experiencia cooperativa de Mondragón», en Homenaje a Del Arco: Del Arco treinta años de vida cooperativa (1973), p. 68.

24 Alejandro Martínez Charterina, o.c., (2015), p. 126. 
aquellos años, a finales de 1843, un pequeño grupo de ellos decidió, entre diversas alternativas que se estudiaron con la idea de mejorar sus condiciones de vida, poner en marcha la Cooperativa de Rochdale (Rochdale Society of Equitables Pioneers) en $1844^{25}$.

Del mismo modo han resuelto satisfactoriamente sus propias crisis de credibilidad de los primeros tiempos, administrativa e ideológica, a las que se refiere Alex Laidlaw en el Informe «las cooperativas en el año 2000»26.

Precisamente esa crisis de identidad, junto con la reclamación de Laidlaw, "las cooperativas deben tratar de mantenerse como islas de cordura en un mundo que se está volviendo loco» ${ }^{27}$, constituye el punto de partida hacia la búsqueda de esa identidad que de fin a la crisis.

Y a partir de entonces se puso en marcha el trabajo que desembocó años después en la Declaración de la Identidad Cooperativa del Congreso Centenario de la Alianza, celebrado en Manchester, en 1995, al que ya nos hemos referido.

$Y$, en consecuencia, las cooperativas realizarán su actividad siguiendo unos principios de funcionamiento 28 , los siete principios cooperativos, asociados justamente a unos valores, los valores cooperativos, que se ponen en práctica a través de ellos $^{29}$, conformándose con los principios y los valores la identidad cooperativa.

Ahora bien, las cooperativas actúan en el mercado donde enfrentan las mismas condiciones del resto de las empresas. Y, en consecuencia, se ven afectadas por las crisis económicas que acompañan la historia de forma recurrente.

Aunque no faltan ejemplos de cooperativas que no han podido salir adelante frente a una crisis ${ }^{30}$, los informes recientes realizados para la OIT por Johnston Birchall y Lou Hammond Ketilson de $2009^{31}$ y de

25 De este modo lo transmite Georges Jacob Holyoake en el capítulo primero de su Historia de los Pioneros de Rochdale (1989), ps. 9-15.

26 Informe que se presentó al Congreso de la Alianza Cooperativa Internacional de Moscú (1980). A. F. Laidlaw, «Las cooperativas en el año 2000»(1982).

27 A. F. Laidlaw, «Las cooperativas...», o.c. (1982), p. 25.

28 Entre ellos la voluntariedad de la asociación, la gestión democrática de la empresa y el reparto no capitalista del beneficio, que caracterizan a la cooperativa frente a las sociedades capitalistas.

29 Recogidos en la Declaración de la Alianza Cooperativa Internacional sobre la Identidad Cooperativa. I.C.A.: Declaración..., o.c., ps 17 y 19.

30 El caso más reciente en nuestro entorno lo constituye la cooperativa Fagor Electrodomésticos. En Alejandro Martínez Charterina, o.c., (2015), p. 127.

31 Johnston Birchall y Lou Hammond Ketilson, Resilience of the Cooperative Business Model in Time of Crisis (2009). 
Johnston Birchall de 201332, ponen de manifiesto la fortaleza de las cooperativas en tiempo de crisis por comparación con otras empresas, hasta el punto de que nuevas cooperativas ocupan espacios que dejan vacíos otras empresas ${ }^{33}$, así como el papel facilitador de servicios a las rentas bajas desempeñado por el crédito cooperativo y su importancia en el sostenimiento de las economías locales, también destacado frente a la banca tradicional ${ }^{34}$.

Esa flexibilidad de las cooperativas, así como su capacidad de resistencia ante la crisis, solo se puede entender desde la consideración de la identidad cooperativa, valores y principios que conforman su modelo empresarial.

\section{Los jóvenes y las cooperativas}

Los jóvenes representan el futuro para cualquier actividad y, en consecuencia, también para las cooperativas los jóvenes de hoy constituyen su futuro. Resulta muy significativa la redacción del quinto principio cooperativo de educación, formación e información, en el Congreso Centenario de Manchester, en 1995:

"Las cooperativas proporcionan educación y formación a los socios, a los representantes elegidos, a los directivos y a los empleados para que puedan contribuir de forma eficaz al desarrollo de sus cooperativas. Informan al gran público, especialmente a los jóvenes y a los líderes de opinión, de la naturaleza y beneficios de la cooperación ${ }^{35}$.

El principio está pidiendo que se informe al gran público de lo bueno que tiene la cooperación. No se trata de guardarlo para los cooperativistas sino de compartirlo. Y de compartirlo especialmente con los jóvenes y los líderes de opinión, con los que mejor van a poder proyectar hacia el futuro la naturaleza y los beneficios de la cooperación.

En la redacción de los principios inmediatamente anterior, la del XXIII Congreso de la $\mathrm{ACI}$ de Viena, en 1966, se recoge al público en general como destinatario de la promoción de la educación, sin mencionar expresamente a los jóvenes ${ }^{36}$.

32 Jonsthon Birchall, Resilience in a downturn: The power of financial cooperatives (2013)

33 Informe Birchall-Ketilson, Resilience..., o.c., (2009), p. 5.

34 Informe Birchall, Resilience in a downturn..., o.c., (2013), p.3.

35 I.C.A.: Declaración de la Alianza... o.c. (1996), p.19.

36 I.C.A.: Report of the ICA Commission on Co-operative Principles, (1967), p. 36. 
Es un hecho que los jóvenes han tenido importancia en la historia del cooperativismo. Con frecuencia se recuerda que veintisiete de los veintiocho pioneros de Rochdale tenían menos de treinta años cuando se fundó la cooperativa. Y desde finales de los años treinta del pasado siglo existía una organización de los jóvenes, Comité Internacional de Jóvenes Cooperativistas, que intensificará su actividad de apoyo al cooperativismo en los años cincuenta, tiempo en que se constituirá la Federación Internacional de Jóvenes Cooperativistas ${ }^{37}$.

Con todo ello, sin embargo, la importancia de los jóvenes para el cooperativismo va a aumentar considerablemente en la parte final del siglo xx así como en los primeros años del presente siglo.

El Congreso XXVIII de la Alianza Cooperativa Internacional, celebrado en Hamburgo, en 1984, adoptó una resolución ${ }^{38}$, a partir de la cual tuvo lugar la Conferencia Mundial de Jóvenes Cooperadores «Participation-Development-Peace: Youth and the Cooperative Idea in a Chanching World», en Varsovia en $1985^{39}$, en la que se atiende y considera la contribución de las cooperativas a los jóvenes tanto en los países en vías de desarrollo, que ya se había destacado anteriormente, cuanto en los países desarrollados, desvinculando esa relación del papel facilitador de la cooperación para el desarrollo y generalizándolo a la institución ${ }^{40}$.

Al mismo tiempo que se celebraba en Manchester el XXXI Congreso Centenario de la Alianza Cooperativa Internacional, en 1995, los jóvenes se reunían en el Colegio Cooperativo en Stanford Hall, Loughborough, en el Tercer Seminario Internacional de Jóvenes, "Young People Co-operation and the Media», para pedir un mejor uso de los medios de comunicación que faciliten las relaciones y contactos entre los jóvenes y las cooperativas ${ }^{41}$.

Precisamente a causa de la importancia que la Alianza Cooperativa Internacional daba a los jóvenes introdujo en su Consejo de Administración un representante de los jóvenes, primero por cooptación, desde 2003, y después, desde 2008, por elección.

37 Laura Gómez, La Alianza Cooperativa Internacional, su desarrollo como institución y en especial como instrumento transformador de la sociedad (1998), ps. 277 y 278.

38 Rita Rhodes and Dionysos Mavrogiannis, Thematic Guide to ICA Congresses 1895 1995. (1995), p. 163.

39 ICA - Supreme Cooperative Council of Poland, World Conference of Young Cooperators, 21-26 october 1985, Warsaw - Poland, Documents and Materials, s.d.

40 Laura Gómez, o.c. (1998), p. 279.

41 En Rita Rhodes and Dionysos Mavrogiannis, o.c., (1995), p, 187, que remite a la Rewiew of International Cooperation, vol 88, n. ${ }^{\circ} 4 / 1995$, ps. 35-38, y Laura Gómez, o.c. (1998), p. 281. 
Como preparación del Año Internacional de las Cooperativas se presentó como tema para el 89. Día Internacional de las Cooperativas, celebrado el 2 de julio de 2011 el de «La juventud, el futuro de las empresas cooperativas», coincidiendo en el tiempo con la celebración del Año Internacional de la Juventud de Naciones Unidas.

En el texto de la Alianza para este acontecimiento se considera la importancia de que las cooperativas atiendan y promuevan la participación de los jóvenes, considerando que el modelo de empresa cooperativa es poco conocido, y que tanto los jóvenes como las cooperativas puedan alcanzar mutuos beneficios de su relación: los jóvenes pueden encontrar empleo en las cooperativas que, siendo empresas democráticas, responsables y éticas, sintonizan con sus inquietudes, y las cooperativas su continuidad con unos jóvenes bien formados profesional y tecnológicamente, y unidos en red ${ }^{42}$.

El año siguiente, 2012, proclamado Año Internacional de las Cooperativas por Naciones Unidas, fue especialmente activo promoviendo los valores y principios cooperativos entre los jóvenes mediante la organización de un concurso de música, video y fotografía.

Por fin, en el Plan para una Década Cooperativa, redactado por Cliff Mills y Will Davies, bajo la orientación del Grupo de Trabajo de Planificación de la Alianza Cooperativa Internacional, aprobado por la Asamblea General de la Alianza, que persigue para el año 2020 que el modelo empresarial cooperativo se reconozca como «líder de la sostenibilidad económica, social y medioambiental», sea «el modelo preferido por la gente», y «el tipo de organización empresarial de más rápido crecimiento» ${ }^{43}$, las referencias a los jóvenes resultan abundantes y centrales.

Siendo el centro de la reflexión la identidad cooperativa, el Plan considera que los jóvenes deben tomar parte tanto en la construcción de esa identidad como en la comunicación de la misma, teniendo en cuenta la importancia de la tecnología y las redes sociales en su forma de comunicarse. Y, concluye en la consiguiente importancia de la educación cooperativa y, en particular, en la formación sobre la identidad cooperativa, para la capacitación de los líderes del futuro44. Y se plantea como uno de los objetivos importantes de la década el de centrarse en los jóvenes para conocer sus mecanismos de crecimiento y relación,

42 Mensaje de la Alianza Cooperativa Internacional en la celebración del 89. Día Internacional de las Cooperativas de $\mathrm{ACl}, 17 .^{\circ}$ Día Internacional de Naciones Unidas de las Cooperativas (2011), La juventud, el futuro de las empresas cooperativas.

43 A.C.I., Plan para una década cooperativa, (2013), p. 3.

44 A.C.I., Plan para una década cooperativa, (2013), ps. 23 y 24. 
así como su capacidad innovadora, para poder diseñar un futuro con su participación ${ }^{45}$.

Además de la atención que prestan las cooperativas y sus organizaciones a la que estamos haciendo referencia, la realidad nos muestra también cómo los jóvenes van poniendo en marcha cooperativas en muchas partes del mundo, construyendo experiencias muy valiosas en términos de información de las dificultades con las que se van encontrando en el camino, así como de las inquietudes y preferencias de los mismos ${ }^{46}$.

\section{Los jóvenes comparten los valores cooperativos}

Desde la perspectiva de cambio acelerado, estado de globalización y crisis con la que hemos comenzado este trabajo, cabe preguntarse en qué medida los jóvenes de hoy comparten unos valores que puedan sintonizar con la identidad cooperativa. Se trata de ver si los jóvenes de hoy muy diferentes de los de las generaciones anteriores pueden encontrar en el cooperativismo el estímulo que ha hecho nacer y desarrollar este modelo de empresa.

Más allá de las intuiciones o creencias particulares podemos disponer de un estudio sobre valores de los jóvenes en España de 2014, y de la aplicación del mismo a los valores cooperativos ${ }^{47}$.

El estudio de referencia divide los jóvenes españoles de 15 a 24 años en 2014 en una tipología de cinco clusters, a los que denomina:

1. Conservadores por la integración.

2. Despreocupados por lo ajeno: instalados en el presente.

45 A.C.I., Plan para una década cooperativa, (2013), ps. 10 y 11

46 En este sentido el libro editado por Julia Smith, Robin Puga, Ian Macpherson, Youth reinventing co-operatives: young perspectives on the international co-operatives movement, (2005), que recoge experiencias de cooperativas de jóvenes y organizaciones de ellas, de muy diversas clases, y procedentes de muchos países, como Canadá, Estados Unidos, Argentina, Colombia, Kenia, Ghana, Uganda, Sudáfrica, Filipinas, Malasia, Japón, India, Indonesia, Serbia, Montenegro y Reino Unido. En nuestro País Vasco comienzan a instalarse las Junior Cooperativas, formadas por estudiantes que tratan de poner en práctica, con apoyo público (dentro del Plan de Reactivación de Empleo aprobado por el Gobierno Vasco) y desde la Universidad de Mondragón, adaptando el modelo finlandés de las Team Learning Cooperatives, los conocimientos que van adquiriendo, para transformarlas en cooperativas de trabajo al final de los estudios.

47 Se trata de la obra de Javier Elzo Imaz y Eusebio Megías Valenzuela (codirectores), et al.: Jóvenes y Valores I: un Ensayo de Tipología (2014), y del artículo de Javier Elzo, «Jóvenes y valores cooperativos», (2015). 
3. Rebeldes con causa y un tanto confusos.

4. Incívicos despreocupados: "Pasotas».

5. ${ }^{\circ}$ Conservador altruista: con escasos recursos y religiosos.

En la tipología se ha tratado de formar clusters muy parecidos dentro de su grupo y muy diferenciados entre los distintos grupos. Los porcentajes atribuidos a los cinco tipos son los siguientes:

$1 .^{\circ} 22,1 \%$

2. $28,2 \%$

3. $21,0 \%$

$4 .^{\circ} 14,7 \%$

$5 .{ }^{\circ} 8,0 \%$

Y en esos cinco tipos se agrupa el 93,7 \%, quedando como «no clasificados» únicamente el 6,3\% ${ }^{48}$.

$Y$, aunque el estudio no hace una referencia expresa a los valores cooperativos se realiza una aplicación específica en la que se destacan dos grupos bien definidos de aquellos tipos en cuyo ámbito puede fructificar la semilla cooperativa frente a aquellos otros en los que no se considera esa posibilidad ${ }^{49}$.

De este modo los clusters $1 .^{\circ}, 3^{\circ}$ y $5 .^{\circ}$, que suman el $51,1 \%$, acogen a jóvenes que están dispuestos a comprometerse, con actitudes proactivas, implicados en lo colectivo, que buscan un cambio respecto al capitalismo financiero actual así como al socialismo real pasado y restante, o que por lo menos buscan la perfección del estado de cosas presente. Al propio tiempo estos tipos $1 .^{\circ}, 3 .^{\circ}$ y $5 .^{\circ}$ presentan tendencias conservadoras desde antes de la crisis o bien como consecuencia de la crisis, «... detestan las trampas, el engaño, el enchufe, la corrupción en suma, abogando por lo honrado, lo leal, lo generoso, lo comprometido, lo solidario, lo tolerante... A la vez aparece... un énfasis en entornos más seguros, una necesidad de orden y seguridad anteriormente no vistas, una rigidificación de las sanciones hacia los comportamientos que amenazan ese orden. ${ }^{50}$

En el otro lado, los clusters $2 .^{\circ}$ y $4 .{ }^{\circ}$, un $42,6 \%$ de los jóvenes, entre ellos lo más jóvenes, se presentan como despreocupados por los

48 Puede verse la disposición de los valores en los tipos, así como las atribuciones porcentuales en el Capítulo 5 «Una tipología de los jóvenes españoles según sus valores» del estudio Javier Elzo Imaz y Eusebio Megías Valenzuela (codirectores), et al. o.c.,(2014), ps. 94 ss. Y una síntesis en Javier Elzo, o.c., (2015), ps. 45 ss.

49 Javier Elzo, o.c., (2015), ps. 55-58.

50 Javier Elzo, o.c., (2015), p. 56. 
demás, y quedan fuera de la capacidad receptiva hacia los valores del cooperativismo.

\section{Conclusión: una mirada al futuro}

A la vista de lo que venimos diciendo tenemos que concluir que hoy, al igual que ayer, a pesar de todos los cambios y las circunstancias particulares, hay un núcleo importante y mayoritario de jóvenes cuyos valores sintonizan con la identidad cooperativa y permiten mirar al futuro con la certeza de que no van a faltar cooperativistas tampoco en esta ocasión. A fin de cuentas, como señala Roberto Rodrigues «El cooperativismo es una filosofía de vida, y de ella están excluidos el individualismo, la ambición personal, la búsqueda del lucro a cualquier costo» ${ }^{51}$.

Son muchos los jóvenes que a través de las cooperativas pueden encontrar sus oportunidades de empleo, disfrute de una vivienda, obtención de servicios diversos, y que pueden mejorar su formación, su capacidad de gestionar y asumir responsabilidades, tanto en los países en vías de desarrollo como en los países desarrollados. Y también las cooperativas pueden recibir las ideas de estos jóvenes, su participación y sus aportaciones en línea con el cambio necesario, su fuerza, su capacidad de sustitución en el liderazgo y en la asunción de responsabilidades. Las cooperativas tienen, en este sentido, que aprovechar a los jóvenes $^{52}$.

Y mirando hacia atrás se debe reivindicar, una vez más, la importancia del principio de educación en las cooperativas, la regla de oro de la que con más frecuencia de lo deseable nos olvidamos, y que aparece llena de sentido para conectar las generaciones sin sobresaltos y dotar de continuidad social a las instituciones a través de las personas.

Por todo ello desde el cooperativismo se debe profundizar en tres aspectos en dirección a los jóvenes a partir del principio de educación.

En primer lugar se debe profundizar en la comunicación. La Alianza Cooperativa Internacional lo explicita con claridad en su Declaración sobre la Identidad Cooperativa, como hemos mencionado anteriormente, al establecer dentro del quinto principio, que las cooperativas «informan al gran público, especialmente a los jóvenes y a los líderes

51 Roberto Rodrigues, siendo Presidente de la $\mathrm{ACl}$, en el Prólogo al libro de José Rodríguez Pérez, Cooperativismo para jóvenes, (2001), p.7.

52 Jo Bibby Scullion «1.Thinking globally», en Julia Smith, Robin Puga, Ian Macpherson (eds.), Youth reinventing co-operatives..., o.c., (2005),ps. 5-7. 
de opinión, de la naturaleza y beneficios de la cooperación» ${ }^{53}$. Se trata de trasladar a la sociedad el convencimiento de las bondades del cooperativismo, especialmente cuando se considera que en todas partes el desconocimiento sobre las cooperativas así como su bajo posicionamiento a pesar de sus éxitos demostrables ${ }^{54}$. Esta comunicación habrá de pensarse para acomodarla a la especificidad de los jóvenes que hoy se mueven en redes de forma directa y participativa.

En segundo lugar se debe profundizar en la formación, la cual permite el acceso al conocimiento, la toma de conciencia y la acción. La formación cooperativa, como campo de autoexpresión, de liderazgo y de democracia, facilita el acoplamiento de la persona a la organización y la asunción de responsabilidades ${ }^{55}$. Frecuentemente se destaca la importancia de la formación cooperativa desde la infancia, desde la escuela, como vehículo de accesibilidad y familiaridad del modelo.

Por fin, en tercer lugar, la participación real de las cooperativas y sus organizaciones para facilitar a los jóvenes tanto el acceso a las cooperativas que ya existen, como la oportunidad de poner en marcha unas nuevas cooperativas de jóvenes ${ }^{56}$.

\section{Bibliografía}

ABADÍA, Leopoldo: El economista esperanzado, Espasa, Barcelona, 2012.

ABADíA, Leopoldo: La crisis ninja y otros misterios de la economía actual, Espasa, Barcelona, 2009.

AbADíA, Leopoldo: La economía en 365 preguntas, Espasa, Barcelona, 2013.

ABADía, Leopoldo: La hora de los sensatos, Espasa, Barcelona, 2010.

AbADía, Leopoldo: ¿Qué hace una persona como tú en una crisis como esta?,

Espasa, Barcelona, 2010.

53 I.C.A.: Declaración de la Alianza... o.c. (1996), p.19.

54 Juan Carlos Mejía Cuarta, "Juventud, ciencia e innovación en el cooperativismo», (2006), p. 82. En la encuesta que recoge el estudio Contribution of Cooperatives and Youth Cooperatives to Youth Employment and Entrepreneurship, del primer trimestre de 2013, se destaca como el mayor desafío a enfrentar la falta de conciencia sobre las cooperativas juntamente con los estereotipos negativos asociados a las cooperativas de jóvenes, a saber, problemas financieros y de gestión.

55 Incluso en otros campos como en la política. Puede verse P.E.Weeraman, Cooperation and Youth, (1973), p. 3.

56 En el País Vasco la Cooperativa Elkar-Lan formada por el Consejo Superior de Cooperativas de Euskadi, la Confederación de Cooperativas de Euskadi y la Federación de Cooperativas de Trabajo Asociado, Enseñanza y Crédito de Euskadi informa, forma, asesora y acompaña en la constitución de nuevas cooperativas, a las que tutoriza y hace seguimiento desde el año 2002, habiendo impulsado en 2015 la constitución de 143 cooperativas que han generado 549 empleos. 
A.C.I.: Plan para una década cooperativa, enero 2013. En www.aciamericas. coop/IMG/pdf/ica_blueprint_es.pdf

ArizMendiARRIETA, José M.a.: «La experiencia cooperativa de Mondragón», en Homenaje a Del Arco: Del Arco treinta años de vida cooperativa, CENEC, Zaragoza, 1973, ps. 65-73.

BIRCHALL, Johnston: Resilience in a downturn: The power of financial cooperatives, ILO, Geneva, 2013.

BIRCHALL, Johnston y KETILSON, Lou Hammond: Resilience of the Cooperative Business Model in Time of Crisis, ILO, Geneva, 2009.

ELzO, Javier: "Jóvenes y valores cooperativos», en en ARNÁEZ ARCE, Vega María (coordinadora): Difusión de los valores y principios cooperativos entre la juventud, Dykinson, Madrid, 2015, ps.41-57.

Elzo IMAZ, Javier y Megías Valenzuela, Eusebio (cdorectores), et al:: Jóvenes y Valores I: un Ensayo de Tipología, Centro Reina Sofía sobre Adolescencia y Juventud Fundación de Ayuda contra la Drogadicción (FAD), Madrid, 2014.

Fauquet, Georges: "Le secteur coopératif», en Oeuvres, Publications de I'Institut des Etudes Coopératives, Paris, 1965, ps. 21-127.

Gómez, Laura: La Alianza Cooperativa Internacional, su desarrollo como institución y en especial como instrumento transformador de la sociedad, Consejo Superior de Cooperativas de Euskadi, Vitoria-Gasteiz, 1998.

HolyOAKE, Georges Jacob: Historia de los Pioneros de Rochdale, Intercoop, Buenos Aires, 1989.

I.C.A.: Declaración de la Alianza Cooperativa Internacional sobre la Identidad Cooperativa. Los principios cooperativos, Consejo Superior de Cooperativas de Euskadi, Vitoria-Gasteiz, 1996.

I.C.A.: Report of the ICA Commission on Co-operative Principles, International Co-operative Alliance, London, 1967.

I.C.A. - Supreme Cooperative Council of Poland: World Conference of Young Cooperators, 21-26 october 1985, Warsaw - Poland, Documents and Materials, s.d.

Informe Oxfam, Gobernar para las élites. Secuestro democrático y desigualdad económica. 20 de enero de 2014. www.oxfamintermon.org/sites/default/ files/documentos /files/bp-working-for-few-political-capture-economic-inequality-200114-es.pdf

Informe Oxfam, La trampa de la austeridad. El verdadero coste de la desigualdad en Europa, septiembre de 2013. www.oxfam.org/sites/www.oxfam. org/files/file_attachments/bp174-cautionary-tale-austerity-inequality-europe-120913-es_3.pdf

Informe temático de Oxfam, Riqueza: Tenerlo todo y querer más, enero de 2015. www.oxfam.org/sites/www.oxfam.org/files/file_attachments/ibwealth-habing-all-wanting-move-190115-es.pdf

Informe Oxfam, Una economía al servicio del 1\%. Acabar con los privilegios y la concentración de poder para frenar la desigualdad extrema, 18 de enero de 2016. www.oxfam.org/sites/www.oxfam.org/files/file_attachments/ bp210-economy-one-percent-tax-havens-180116-es_0.pdf

KRUGMan, Paul: ¡Acabad ya con esta crisis!, Crítica, Barcelona, 2012. 
Martínez Charterina, Alejandro: Introducción a la economía, Dykinson, Madrid, 2011.

Martinez ChARTERINA, Alejandro: "Las cooperativas frente a la crisis», en Boletín de la asociación Internacional de Derecho Cooperativo, n. ${ }^{\circ} 44$, Universidad de Deusto, Bilbao, 2010, ps. 195-219.

Martínez ChARTERINA, Alejandro: "Las cooperativas y los jóvenes en la actualidad», en Arnáez ArCE, Vega María (coordinadora): Difusión de los valores y principios cooperativos entre la juventud, Dykinson, Madrid, 2015, ps. 121134.

MejíA CuARTA, Juan Carlos: "Juventud, ciencia e innovación en el cooperativismo», en Revista de la Cooperación Internacional, volumen 39, n. ${ }^{\circ}$, edición en español a cargo de INTERCOOP, Buenos Aires, 2006, ps. 73-82.

Mensaje de la Alianza Cooperativa Internacional (2011), La juventud, el futuro de las empresas cooperativas. En www.aciamericas.coop/lMG/pdf/diainternacional2011.pdf

Rhodes, Rita and Mavrogiannis, Dionysos: Thematic Guide to ICA Congresses1895-1995, ICA, Geneva, 1995.

Rodríguez Pérez, José: Cooperativismo para jóvenes, Editorial Lazos Cooperativos, Buenos Aires, 2001.

SeRRES, Michel: Pulgarcita, Editorial Gedisa, Barcelona, 2014. (El original, Petite poucettte, Éditions Le Pommier, 2012).

SMITH, Julia, PUGA, Robin, MACPHERSON, Ian (eds.): Youth reinventing co-operatives: young perspectives on the international co-operative movement, British Columbia Institute for Co-operative Studies, Victoria, 2005.

Weeraman, P.E.: Cooperation and Youth, I.C.A., New Delhi, 1973. 